\section{SIRT1 and anxiety}

The ability to manage anxiety, an emotion expressed in threatening or challenging conditions, is necessary for navigating new environments. Treatments for anxiety disorders increase serotonin and norepinephrine levels by inhibiting monoamine oxidases and selective serotonin reuptake. Now, Leonard Guarente and colleagues report that SIRT1, a nicotinamide adenine dinucleotide $\left(\mathrm{NAD}^{+}\right)$-dependent protein deacetylase, influences anxiety and exploratory behavior by increasing transcription of the Maoa gene encoding monoamine oxidase A (Cell 147, 1459-1472, 2011). Mice with a brain-specific knockout of Sirt1 had higher levels of exploratory behavior than wild-type littermates. In contrast, mice that overexpressed Sirt1 had lower levels of exploratory behavior. Knockout mice were more resistant to depression in a 'social defeat' paradigm in which depression manifests in experimental male mice after multiple exposures to more dominating males. Knockout mice also had higher levels of serotonin and noradrenalin but lower levels of 5-hydroxyindoleacetic acid (5-HLAA) than wild-type littermates. MAO-A converts serotonin into 5-H1AA, and Maoa mRNA levels were lower in Sirt1-null mice and higher in Sirt1-overexpressing mice than in wild-type littermates, respectively. Luciferase reporter assays suggested that SIRT1 is needed to directly activate the Maoa promoter. Further experiments showed that SIRT1 deacetylates the NHLH2 transcription factor, which directly modulates the Maoa promoter.

\section{Gregarious locusts}

Desert locusts alternate between solitary and gregarious lifestyles. In the solitary phase, locusts avoid conspecifics, have cryptic appearance and behavior, move slowly and eat restricted diets. In contrast, gregarious locusts are active, have bright color and attract other locusts. Phase changes are regulated by population density and are an adaptation to the desert environment where rain is unpredictable. Gregarious locusts are notorious pests, as they can accumulate into devastating swarms. Now, Jozef Vanden Broeck and colleagues report a central role for protein kinase A (PKA) in the transition from solitary to gregarious behavior in locusts (Proc. Natl. Acad. Sci. USA, published online 19 December 2011; doi:10.1073/pnas.1114990109). Within 4 h of crowding, solitary locusts attain several key characteristics of gregarious behavior. The authors found that solitary locusts treated with a PKA inhibitor, KT5720, remained solitary after crowding. The authors performed RNA interference (RNAi)-mediated knockdown of a regulatory R1 subunit of PKA, which normally leads to the inhibition of PKA function, and found evidence of increased gregarious behavior. Injecting already-gregarious locusts with the KT5720 inhibitor did not induce a change to solitary behavior, which shows that gregarious behavior does not depend on PKA activity. Instead, these findings suggest a role for PKA in learning, or acquiring, gregarious behavior.

\section{DICER 1 in ovarian cancers}

Germline mutations in DICER1 underlie a rare syndrome associated with susceptibility to pleuropulmonary blastoma, cystic nephroma and ovarian sex-cord stromal tumors. David Huntsman, Gregg Morin and colleagues (N. Engl. J. Med., published online 21 December 2011; doi:10.1056/ NEJMoa1102903) now show that somatic mutations in DICER1 occur at high frequency in non-epithelial ovarian cancers. The authors per-

Written by Orli Bahcall, Pamela Feliciano, Wayne Peng \& Kyle Vogan formed transcriptome and exome sequencing in two Sertoli-Leydig cell tumors, four juvenile granulosa cell tumors and eight primitive germ cell tumors and identified four missense mutations in DICER1 affecting the metal-binding region of the RNase IIIb domain. They subsequently sequenced the portion of DICER 1 encoding this region in 101 additional non-epithelial ovarian tumors and identified somatic mutations in $60 \%$ of Sertoli-Leydig cell tumors. They also found similar mutations in 1 of 14 nonseminomatous testicular germ cell tumors and in 2 of 5 embryonal rhabdomyosarcomas. Functional studies showed that these mutations impair RNase IIIb activity but do not affect the activity of RNase IIIa. On the basis of these findings, the authors propose that these mutations result in an oncogenic miRNA profile whose pathogenicity may be restricted to specific cell types or developmental settings, thereby accounting for the high prevalence of these mutations in particular tumor types.

$K V$

\section{Genomics of endangered primates}

Yoav Gilad and colleagues report an RNA-seq analysis combined with de novo gene assembly for 16 mammalian species, including humans and 11 nonhuman primates (Genome Res., published online 29 December 2011; doi:10.1101/gr.130468.111). They sequenced the RNA from liver from each of these species. Because many of these species have no sequenced genome, they developed a new algorithm using a de Bruijn graph-based approach for de novo assembly and alignment of gene sequences across species. They characterized levels of genetic diversity and estimated phylogenies based either on gene sequences or gene expression, which reassuringly recapitulated the known primate phylogeny. They found evidence of positive selection on 499 candidate genes, with an enrichment for peroxisomal genes among the top-ranked genes. As many of the included species are on the International Union for Conservation of Nature (IUCN) endangered list, they examined genetic diversity by conservation status but found no clear relationship. Interestingly, the highest genetic diversity was found in the two most endangered species in the study, the blackand-white ruffed lemur and Coquerel's sifaka. This report presents the first genome-scale datasets and genetic diversity estimates for many of these species, providing a resource for comparative genomic studies and ongoing conservation efforts.

$O B$

\section{Cardiac symptoms of Rett syndrome}

The genetic link between mutations in the MECP2 gene encoding the methyl-CpG-binding protein and Rett syndrome (RTT), an X-linked neurodevelopmental disorder affecting approximately 1 in 10,000 girls, was first reported in this journal (Nat. Genet. 23, 185-188, 1999). Although the MeCP2-mediated epigenetic mechanism causing neurological symptoms in RTT has been well studied, little is known about the etiology of cardiorespiratory symptoms of RTT, which contribute to a high incidence of sudden unexpected death. Jeffrey Neul and colleagues report that $18.5 \%$ of individuals with RTT show prolonged QT components (long-QT) in their electrocardiograms (Sci. Transl. Med. 3, 113ra125, 2011). The authors examined Mecp2-null mice and observed long-QT, tachycardia and cardiac arrhythmia in young hemizygous males and older heterozygous females. Nervous system-specific ablation of Mecp2 led to the same phenotypes. Long-QT symptom results from slow repolarization of the autonomic innervation to the heart. The authors show that the cardiac phenotype of Mecp2-null mice can be rescued by a sodium channel blocker but not by a $\beta$-adrenergic receptor antagonist, suggesting delayed sodium channel closure as the disease mechanism. 